\title{
TRANSITIONAL DISKS
}

\section{I can see your halo, halo, halo}

Using the Atacama Large Millimeter/ submillimeter Array (ALMA), Ruobing Dong and collaborators (Astrophys. J., in the press; preprint at https://arxiv.org/abs/1701.05189) have imaged three isotopologues of carbon monoxide and the $1.3 \mathrm{~mm}$ dust continuum emission in the transitional disk J160421.7-213028 (pictured). The cavity or gap that defines this disk as transitional is clearly visible with ALMA's $0.25^{\prime \prime}$ spatial resolution, which at the distance of the disk is $\sim 35$ au. Combined with an $\mathrm{H}$-band scattered light image (of wavelength 1.5-1.8 $\mu \mathrm{m}$; not shown), the authors are able to identify the distributions of three of the main components of this transitional disk: sub-micrometre-sized (small) dust, millimetre-sized (large) dust and the molecular gas.

Both dust populations indicate a sharp transition in dust density at a

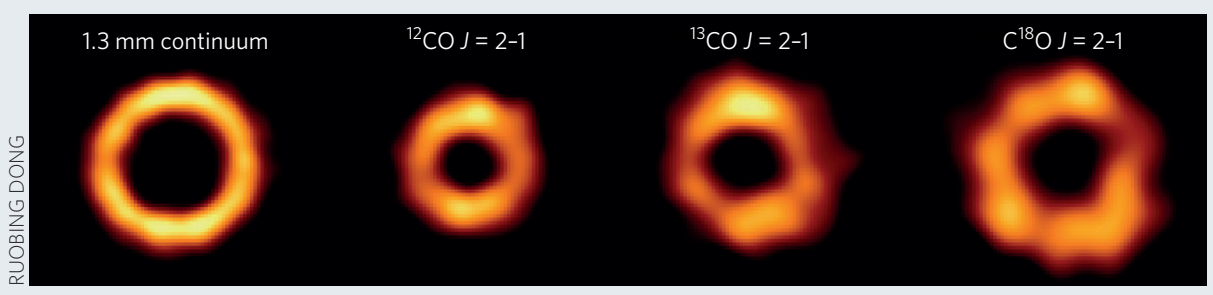

radius of $\sim 70$ au, with the cavity at least 1,000 times less concentrated in dust than the surrounding disk. In the molecular gas observations, the cavity is significantly smaller, extending only 15 au from the central star. Its edge is also more gradual, with the surface density increasing steadily between 15 and 70 au.

The cause of the central cavities in transitional disks is still an open question. A leading theory is that orbiting planets might sculpt a cavity opening through tidal interactions with the disk material. A single planet might open up a gap (such as those seen in the gas-rich disk of TW Hydrae) and multiple planets might open up a cavity like the one seen here. Alternatively, strong radiation from the star might indirectly 'blow' inner material away in a process called photoevaporation. This can cause a cavity to form if the disk accretion rate is sufficiently small. In this scenario, however, the inner edges of both the dust disk and the gas disk are sharply defined.

PAUL WOODS 